\title{
ON THE RELATIVE EXTREMA OF THE TURAN EXPRESSION FOR BESSEL FUNCTIONS
}

\author{
By S. K. Lakshmana Rao \\ (Department of Applied Mathematics, Indian Institute of Science, Bangalore-12) \\ Received October 27, 1960 \\ (Communicated by Professor B. S. Madhava Rao, F.A.sc.)
}

Some years ago Otto Szász ${ }^{1}$ showed that the Bessel function $\mathrm{J}_{n}(x)$ satisfies the inequality

$$
\begin{gathered}
\Delta_{n}(x) \equiv\left[\mathrm{J}_{n}(x)\right]^{2}-\mathrm{J}_{n+1}(x) \mathrm{J}_{n-1}(x) \geq \frac{1}{n+1}\left[\mathrm{~J}_{n}(x)\right]^{2}, \\
n \geq 0, \quad x \text { real }
\end{gathered}
$$

wherefrom he deduced the interesting inequality

$$
\triangle_{n}(x)>0 \text { for } n>0, x \text { real. }
$$

The Turán inequality (2) for the function $\mathrm{J}_{n}(x)$ is an immediate consequence of the identity ${ }^{2}$

$$
\Delta_{n}(x)=\frac{4}{x^{2}} \sum_{k=0}^{\infty}(n+2 k+1)\left(\mathrm{J}_{n+2 k+1}(x)\right)^{2}
$$

which represents $\Delta_{n}(x)$ as a series of positive quantities whenever $n \geq-1$. V. R. Thiruvenkatachar and T. S. Nanjundiah ${ }^{3}$ obtained a new positive representation for $\Delta_{n}(x)$ by proving the identity

$$
\begin{aligned}
\Delta n(x)= & \frac{1}{n+1}\left(\mathrm{~J}_{n}(x)\right)^{2}+\frac{2}{n+2}\left(\mathrm{~J}_{n+2}(x)\right)^{2} \\
& +2 n \sum_{k=2}^{\infty}[(n+k-1)(n+k+1)]^{-1}\left(\mathrm{~J}_{n+k}(x)\right)^{2}
\end{aligned}
$$

and the above result (1) of Otto Szász follows from this immediately.

While the above representations for $\Delta_{n}(x)$ are quite interesting we may also proceed to deduce the inequality (2) by considering the behaviour of the function $\Delta_{n}(x)$ itself. This procedure leads to several questions concerning the sequences of relative maxima and minima of $\Delta_{n}(x)$ and the

\footnotetext{
* Present address: Regional Engineering College, Warangal (A.P.), India.
} 
aim of the present note is the study of these sequences. We prove in particular (Theorem 4 below) that the $r$-th relative maximum of $\Delta_{n}(x)$ is larger than the $r$-th relative maximum of $\Delta_{n+1}(x)$, for any fixed value of $r$. This answers the question first raised by John Todd for the classical orthogonal polynomials and settled for the Hermite function $\mathrm{H}_{n}(x)$ by Otto Szász ${ }^{4}$ and for the Turán expression for the Hermite function by the author. ${ }^{5}$

THeorem 1: When $n>0$, the relative maxima of $\Delta_{n}(x)$ occur at the zeros of $\mathbf{J}_{n-1}(x)$ and the relative minima occur at the zeros of $\mathbf{J}_{n+1}(x)$.

On differentiating the expression

$$
\triangle_{n}(x)=\left(\mathrm{J}_{n}(x)\right)^{2}-\mathrm{J}_{n+1}(x) \mathrm{J}_{n-1}(x)
$$

twice, we get

$$
\frac{d}{d x} \triangle_{n}(x)=\frac{2}{x} \mathrm{~J}_{n+1}(x) \mathrm{J}_{n-1}(x)
$$

and

$$
\begin{gathered}
\frac{d^{2}}{d x^{2}} \triangle_{n}(x)=\frac{2}{x} \mathbf{J}_{n+1}(x) \frac{d}{d x} \mathbf{J}_{n-1}(x)+\frac{2}{x} \mathrm{~J}_{n-1}(x) \frac{d}{d x} \mathbf{J}_{n+1}(x) \\
-\frac{2}{x^{2}} \mathrm{~J}_{n+1}(x) \mathrm{J}_{n-1}(x)
\end{gathered}
$$

Let $0<x_{1, s}<x_{2, s}<\ldots$ denote the zeros of $J_{s}(x)$ in the ascending order. From (7) we see that

$$
\left(\frac{d^{2}}{d x^{2}} \Delta_{n}(x)\right)_{x^{n} x_{r, n-1}}=-4 n\left\{\left(\frac{1}{x} \mathbf{J}_{n}(x)\right)^{2}\right\}_{x_{=x} x_{r, n-1}}<0
$$

and

$$
\left(\frac{d^{2}}{d x^{2}} \Delta_{n}(x)\right)_{x=x_{r, n+1}}=4 n\left\{\left(\frac{1}{x} \mathbf{J}_{n}(x)\right)^{2}\right\}_{x=x_{r, n+1}}>0 .
$$

Hence the theorem.

The statement in the above theorem is reversed if $n<0$. In what follows we assume that $n>0$.

If we denote the successive relative maxima of $\Delta_{n}(x)$ by $\mathrm{M}_{1, n}$, $\mathbf{M}_{2, n}, \ldots$ and the successive relative minima by $m_{1, n}, m_{2, n}, \ldots$ respectively, we have

$$
\mathrm{M}_{r, n}=\triangle_{n}\left(x_{r, n-1}\right)=\left(\mathrm{J}_{n}\left(x_{r, n-1}\right)\right)^{2},
$$


and

$$
m_{r, n}=\Delta_{n}\left(x_{r, n+1}\right)=\left(\mathrm{J}_{n}\left(x_{r, n+1}\right)\right)^{2} .
$$

We may also observe incidentally that

$$
m_{r, n-1}=\left(\mathrm{J}_{n-1}\left(x_{r, n}\right)\right)^{2}=\left(\mathrm{J}_{n+1}\left(x_{r, n}\right)\right)^{2}=\mathrm{M}_{r, n+1} .
$$

Since the quantities $\mathrm{M}_{r, n}$ and $m_{r, n}$ are positive for all values of $n$, it follows that

$$
\triangle_{n}(x)>0
$$

which is Turán's inequality for the Bessel function $\mathrm{J}_{n}(x)$.

THEOREM 2: The sequences of relative maxima and relative minima of $\Delta_{n}(x)$ are decreasing beyond a certain value of $r$. More specifically

$$
\mathbf{M}_{r, n}>\mathbf{M}_{r+1, n} \text { if } x_{r, n-1}>\xi=\sqrt{2 n(n-2)}
$$

and

$$
m_{r, n}>m_{r+1, n} \text { if } x_{r, n+1}>\eta=\sqrt{2 n(n+2)} .
$$

If $0<n \leq 2$, we define $\xi=0$.

Consider the function

$$
f(x)=\Delta n(x)+\frac{2 n}{x^{2}}\left(\mathrm{~J}_{n-1}(x)\right)^{2}
$$

Then, by differentiation we get

$$
\begin{aligned}
f^{\prime}(x) & =\frac{2}{x} \mathrm{~J}_{n+1}(x) \mathrm{J}_{n-1}(x)-\frac{4 n}{x^{3}}\left(\mathrm{~J}_{n-1}(x)\right)^{2}+\frac{4 n}{x^{2}} \mathrm{~J}_{n-1}(x) \frac{d}{d x} \mathbf{J}_{n-1}(x) \\
& =\frac{2}{x^{3}}\left(2 n(n-2)-x^{2}\right)\left(\mathrm{J}_{n-1}(x)\right)^{2}
\end{aligned}
$$

so that $f(x)$ is increasing in $0<x<\xi$ and decreasing in $x>\xi$. Also at the relative maxima of $\Delta_{n}(x), f(x)=\Delta_{n}(x)$ and hence the assertion that $\mathbf{M}_{r, n}>\mathbf{M}_{r+1, n}$ if $x_{r+1, n-1}>x_{r, n-1}>\xi$. To prove the corresponding result for the relative minimum, consider the function

$$
g(x)=\Delta n(x)-\frac{2 n}{x^{2}}\left(\mathrm{~J}_{n+1}(x)\right)^{2} .
$$

Differentiating this we have

$$
g^{\prime}(x)=\frac{2}{x^{3}}\left(2 n(n+2)-x^{2}\right)\left(\mathbf{J}_{n+1}(x)\right)^{2}
$$


so that $g(x)$ is decreasing in $x>\eta$. Since $g(x)$ coincides with $\Delta_{\mathfrak{n}}(x)$ at all the relative minima of $\Delta_{n}(x)$, it follows that $m_{r, n}>m_{r+1, n}$ if $x_{r+1, n+1}>$ $x_{r, n+1}>\eta$.

This completes the proof of the theorem.

THeOREM 3: The $r$-th relative maximum of $\Delta_{n}(x)$ is greater than the $r$-th relative minimum, i.e., $\mathrm{M}_{r, n}>m_{r, n}$.

We have $\Delta_{n}\left(x_{r, n-1}\right)=\mathbf{M}_{r, n}$ and $\Delta_{n}\left(x_{r, n+1}\right)=m_{r, n}$ and $(d / d x) \Delta_{n}(x)$ $=(2 / x) \mathrm{J}_{n+1}(x) \mathrm{J}_{n-1}(x)$. From the interlacing properties of the zeros of Bessel functions we know that ${ }^{6,7}$

$$
x_{r-1, n+1}<x_{r, n-1}<x_{r, n}<x_{r, n+1}<x_{r+1, n-1} .
$$

Hence in the interval $\left(x_{r, n-1}, x_{r, n+1}\right), \operatorname{Sgn}(d / d x) \Delta_{n}(x)=(-1)^{2 r-1}=-1$, so that $\Delta_{n}(x)$ is decreasing in the interval and $\Delta_{n}\left(x_{r, n-1}\right)>\Delta_{n}\left(x_{r, n+1}\right)$ or $\mathbf{M}_{r, n}>m_{r, n}$ which proves the theorem.

By employing the functions $f(x)$ and $g(x)$ already introduced, we can show further that

$$
\mathrm{M}_{r, n}>\left(1+\frac{8 n^{3}}{x_{r, n+1}^{4}}\right) m_{r, n} \text { if } x_{r, n-1}>\xi
$$

and

$$
\mathbf{M}_{r, n}>\left(1-\frac{8 n^{3}}{x_{r, n-1}^{4}}\right)^{-1} m_{r, n} \text { if } x_{r, n-1}>\eta .
$$

THEOREM 4: For a fixed value of $r$, the $r$-th relative maxima of $\Delta_{n}(x)$ form a sequence of decreasing functions of $n$, i.e., $\mathbf{M}_{r, n}>\mathbf{M}_{r, n+1}$. We have already noticed that $\Delta_{n}\left(x_{r, n-1}\right)=\mathbf{M}_{r, n}$. We may see easily that $\mathrm{M}_{r, n+1}=\Delta_{n+1}\left(x_{r, n}\right)=\Delta_{n}\left(x_{r, n}\right)$. Also from the relation $(d / d x) \Delta_{n}(x)$ $=(2 / x) \mathrm{J}_{n+1}(x) \mathrm{J}_{n-1}(x)$, it follows that in the interval $\left(x_{r, n-1}, x_{r, n}\right)$, Sgn $(d / d x) \triangle_{n}(x)=-1$, so that $\Delta_{n}(x)$ is decreasing in the interval and $\Delta n\left(x_{r, n-1}\right)>\Delta n\left(x_{r, n}\right)$. Whence the theorem.

Analogously for the relative minima, we have

THEOREM 5: For a fixed value of $r$, the $r$-th relative minima of $\Delta_{n}(x)$ form a sequence of decreasing functions of $n$, i.e., $m_{r, n}>m_{r, n+1}$.

We have noticed earlier that $m_{r, n-1}=\mathrm{M}_{r, n+1}$. Combining this with the result of the previous theorem, we see that $m_{r, n-1}>m_{r, n}$ which proves the assertion. 


\section{REFERENCES}

1. Otto Szász

2. Watson, G. N.

3. Thiruvenkatachar, V. R. and Nanjundiah, T. S.

4. Otto Szász
.. "Inequalities concerning ultraspherical polynomials and Besse! functions," Proceedings of the American Mathematical Society, 1950, 1, 256-67.

.. A Treatise on the Theory of Bessel Functions, 2nd Edition, Cambridge, 1948, p. 152.

"Inequalities concerning Bessel functions and orthogonal polynomials," Proc. Ind. Acad. Sci., 1951, 33 A, 373-84.

.. "On the telative extrema of the Hermite orthogonal functions," Jour. Ind. Math. Soc. (N.S.), 1951, 15, 129-34.

5. Lakshmana Rao, S. K. .. "On the relative extrema of the Turán expression for the general Hermite function," Indian Institute of Science Golden Jubilee Research Volume, 1959, pp. 200-04.

6. Watson, G. N.

.. "Ref. (2) above, p. 480.

7. Gray, A.: Mathews, G. B. A Treatise on Bessel Functions, 1952, p. 242. and Mac Robert. T. M. 